\title{
FACTORES DETERMINANTES DEL DUELO EN PERSONAS CON DISCAPACIDAD INTELECTUAL Y TEA: REVISIÓN SISTEMÁTICA
}

\section{Determinants of Grief in People with Intellectual Disabilities and ASD: Systematic Review}

\author{
Marta Bóveda Hermosilla \\ Universidad de Salamanca. Facultad de Psicología \\ martaboveda1997@usal.es \\ Noelia Flores RobAinA \\ Universidad de Salamanca. Facultad de Psicología
}

Recepción: 12 de octubre de 2020

Aceptación: 1 de febrero de 2021

Resumen: Antecedentes: Históricamente las personas con discapacidad han sido apartadas de los temas relacionados con la muerte y el duelo. Sin embargo, existen factores que afectan e influyen en el afrontamiento que realizan ante una pérdida. Se precisa conocer cómo experimentan el duelo las personas con discapacidad intelectual y discapacidades del desarrollo con objeto de mejorar la atención ofrecida al colectivo. Método: Se ha llevado a cabo una revisión sistemática de los artículos científicos publicados durante los años 2013 a 2020 en las principales bases de datos. De 585 artículos identificados se seleccionaron 14 que cumplieron los criterios de inclusión. Resultados: La inclusión de las personas con discapacidad en los rituales funerarios, mejorar la comunicación y facilitar la expresión emocional se consideran aspectos esenciales para un adecuado afrontamiento del duelo. Conclusiones: Es necesario ofrecer apoyos adaptados al tipo de discapacidad, incrementar la participación y enseñar estrategias de afrontamiento adaptativas en los temas relativos a la muerte y el duelo. Para ello se precisa incrementar la investigación aplicada en aras de diseñar y poner en marcha programas de educación para la muerte y de intervención psicológica ajustados a las necesidades que presenta este colectivo. 
Palabras clave: discapacidad intelectual; trastorno del espectro del autismo; duelo; muerte; revisión sistemática.

AвSTRAct: Background: Historically, people with disabilities have been excluded from issues related to death and grief. However, there are factors that affect and influence their coping with a loss. It is necessary to know how people with intellectual and developmental disabilities experience grief in order to improve the care offered to the group. Method: A systematic review of the scientific articles published during the years 2013 to 2020 in the main databases was carried out. Of 585 articles identified, 14 were selected that met the inclusion criteria. Results: Including people with disabilities in funeral rituals, improving communication and facilitating emotional expression are essential aspects for adequate coping with grief. Conclusions: It is necessary to offer supports adapted to the type of disability, increase participation and teach adaptive coping strategies on issues related to death and grief. For this, it is necessary to increase applied research in order to design and implement educational programs for death and psychological intervention adjusted to the needs of this group.

KEYWORDS: intellectual disability; autism spectrum disorder; grief; death; systematic review.

\section{Introducción}

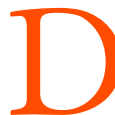

URANTE LAS ÚLTIMAS DÉCADAS, ha aumentado considerablemente la esperanza de vida debido al cambio en las tendencias y las proyecciones demográficas. Los avances tecnológicos y médicos también han contribuido a incrementar los años de vida en las personas ancianas o con algún tipo de enfermedad, prolongando de esta forma la etapa final de la vida (McBride y Simms, 2001). Este aumento en la esperanza de vida provoca que las personas vivan más años, al igual que les ocurre a las personas con discapacidad intelectual (a partir de ahora DI) y otras discapacidades del desarrollo, quienes, debido a este incremento, es probable que tengan que enfrentarse a un proceso de duelo por la pérdida de un familiar, cuidador o ser querido. Pese a ello, en muchas ocasiones, las personas con discapacidades intelectuales y del desarrollo son excluidas de estos procesos porque los familiares piensan que no van a comprender el significado de la muerte o incluso que les afectaría muy negativamente (Bergman et al., 2010; Blackman, 2008; Machale y Carey, 2002), privándoles así de su derecho a experimentar duelo. Este hecho parece ser fruto de la desinformación existente, pues no abundan las investigaciones que hayan abordado este tema en el ámbito de la DI y menos aún en los trastornos del desarrollo, como es el caso de las personas con trastornos del espectro del autismo (a partir de ahora TEA). Sin embargo, diferentes autores aluden a que la inclusión y la participación de estas personas en las ceremonias y rituales funerarios, así como recibir formación en temas relacionados con la muerte, podría ayudar al individuo a afrontar, de manera más saludable, este hecho inevitable de la vida (Dodd et al., 2005). La educación para la muerte, también denominada "pedagogía de la muerte”, tiene que ver con la enseñanza didáctica sobre 
la muerte como una experiencia vital cuyo abordaje incluye valores y herramientas para abordar los momentos de pérdida (Wass y Corr, 1982). Se pretende así facilitar la comprensión de la persona respecto a la universalidad de este hecho, así como trabajar sus emociones y dotarla de estrategias que le permitan afrontar esta experiencia e integrarla y normalizarla como parte de la vida. Todo ello permitirá que la persona tenga conocimientos que le sirvan de preparación ante duelos futuros. En el ámbito de la discapacidad intelectual, las escasas investigaciones realizadas sobre experiencias de pedagogía de la muerte con estas personas han demostrado su utilidad a la hora de ayudarlas a adquirir e interiorizar conceptos relacionados con la muerte, lo que repercute en una mayor disposición de la persona a la hora de hablar sobre la pérdida, la muerte y el duelo (Rodríguez-Herrero et al., 2013). Cuestiones, sin embargo, que aún precisan ser investigadas con mayor profundidad debido a las limitadas evidencias que existen en este ámbito.

No obstante, en los últimos años se está haciendo un esfuerzo importante por contribuir a aportar estudios empíricos y revisiones sobre el estado de la investigación en este campo (Cristóbal et al., 2017; Dowling et al., 2006; Lord et al., 2017).

No cabe duda de que la muerte es un hecho natural y esperado por el que todas las personas vamos a pasar en la vida. El duelo que produce dicha muerte se define como la reacción emocional, psicológica, cognitiva y comportamental producida a partir de una pérdida de la persona significativa (Barreto et al., 2012). Los síntomas que puede experimentar cada persona son diversos y van desde el estado de ánimo depresivo, a la ansiedad, pena o angustia emocional o hasta enfermedades físicas, dependiendo de la forma en que se afronte el proceso de duelo. De tal manera que es posible distinguir entre dos tipos del duelo: el duelo común o normal, caracterizado por el aumento de la angustia en el momento de la pérdida y una posterior recuperación en ausencia de patología, y el duelo crónico, complicado o patológico, caracterizado por sentimientos intensos de angustia antes, durante y después de que se produzca la pérdida, que persisten durante años más allá de la pérdida y que puede generar serias consecuencias para la salud mental (p. e. trastornos depresivos, ansiedad, insomnio crónico, etc.).

A menudo se tiende a asumir y argumentar erróneamente que las personas con DI, con independencia de su edad o grado de afectación, no son capaces de entender la finalidad, irreversibilidad e inevitabilidad de la muerte (Dodd et al., 2005; Stoddart et al., 2002). Este tipo de pensamientos desacertados provocan que, en una gran parte de los casos y con la sana intención de ayudar o proteger a la persona con DI, se actúe de manera equivocada y se limite la posibilidad de que estas puedan participar en los procesos relacionados con la muerte y experimentar también los sentimientos y las reacciones más comunes asociados a la pérdida de un ser querido. Dicha privación del duelo puede conllevar consecuencias aún más negativas para la persona con DI y generar, a largo plazo, un proceso de duelo complicado o patológico (Dodd et al., 2005). Por tanto, involucrar más a estas personas y ayudarles en la comprensión y afrontamiento de la muerte y los sentimientos asociados con ella se consideran una de las actuaciones a acometer en beneficio de este colectivo. Así, en el estudio realizado por McEvoy et al. (2012) concluyeron que, en las personas adultas con DI, se daba una falta de comprensión de lo que supone el envejecimiento y la muerte como parte 
natural e inevitable del ciclo de la vida. Ello, según los autores, parecía deberse al trato infantilizado que solían ofrecer los familiares y cuidadores de las personas con DI ya que, con objeto de evitarles sufrimiento, no se les animaban a actuar, participar o realizar las actividades apropiadas para su edad (Dodd et al., 2005).

No cabe duda de que, para las personas con DI que tienen un fuerte vínculo o dependencia de su cuidador principal, su muerte puede suponer un suceso traumático que afectará a su funcionamiento diario, por lo que debe ser tratado y abordado de forma adecuada (Delorme, 1999). Numerosas investigaciones afirman que las personas con DI y del desarrollo suelen quedar apartadas de los procesos de duelo (Brelstaff, 1984; Cathcart, 1995; Hollins y Esterhuyzen, 1997; Mappin y Hanlon, 2005), pero apenas hay estudios en esta población que aporten datos sobre las personas que sí que son partícipes de estos procesos y de las intervenciones o actuaciones que se llevan a cabo con ellos para vivir la situación de la mejor manera posible. Un ejemplo de los escasos trabajos al respecto es el estudio realizado por Machale et al. (2009), donde evidenciaron que las personas adultas con DI son capaces de comprender lo que supone la muerte en cuanto a su finalidad, irreversibilidad y universalidad. Esto nos permite afirmar que las personas con DI son capaces de comprender lo que supone la muerte y que, por tanto, pueden y deben pasar por un proceso de duelo cuando un ser querido fallece. La forma en que la persona con DI viva ese proceso de duelo dependerá, entre otros, de la forma en que pueda expresar sus sentimientos o emociones. En este sentido, cuando las reacciones posteriores al duelo se internalizan, es probable que aparezcan síntomas de tristeza o depresión, que en muchas ocasiones son difíciles de detectar. Por otro lado, esos sentimientos también pueden externalizarse, por ejemplo, a través del comportamiento desafiante, que puede ser atribuido a la discapacidad en vez de al proceso de duelo.

Al igual que las personas con DI son “apartadas” de los rituales de muerte, ocurre lo mismo en las personas con discapacidades del desarrollo. Por lo general, se tiende a asumir que estas personas tampoco serán capaces de comprender la muerte, limitando así su capacidad emocional y equiparando una capacidad cognitiva más limitada con una capacidad emocional también más limitada, provocando que se ignore y se les prive, en consecuencia, de la gestión del dolor. Todas estas suposiciones han sido cuestionadas en la literatura científica por autores como Kitching (1987), Oswin (1992) o James (1995). Se argumenta que una de las causas por las que suelen ser excluidas del proceso de duelo es porque tienen dificultades en la expresión y comprensión de sentimientos. Por tanto, si no se realiza una intervención correcta en la gestión de estos sentimientos, la experiencia de la pérdida podrá ser aún más complicada. Además, es probable que esta exclusión o prohibición aumente el riesgo de sufrir problemas psicológicos futuros, como puede ser el desarrollo de un duelo complicado, ya que no se está satisfaciendo la necesidad de ayuda adicional que dichas personas precisan.

Por lo tanto, hay que incrementar la investigación pues aún es muy limitado el número de estudios sobre este tema. Comprobado esto, se plantea otra cuestión más, ¿experimentan las personas con TEA un proceso de duelo?

Debido a la escasa literatura existente, es difícil conocer con exactitud cómo experimentan las personas con TEA estos procesos. Los estudios realizados afirman que 
suelen tener dificultades en la comprensión del lenguaje y los conceptos abstractos, por lo que, al mantener una conversación relacionada con la pérdida de un ser querido, puede ser difícil de comprender para ellos por implicar términos o expresiones abstractas (Mesibov et al., 2005). Además, sus dificultades en las relaciones sociales y en la comunicación pueden inhibir su capacidad para conectar y empatizar con el dolor que sufren las demás personas y para expresar sus sentimientos. Esto puede perjudicarles perdiendo la comunidad de apoyo que se forma durante el momento de la pérdida. Diferentes trabajos han señalado que las personas con TEA tienen más riesgo de pasar por un duelo complicado en comparación con las personas con un desarrollo típico (Hume et al., 2016; Melhem et al., 2007).

Las investigaciones realizadas sobre el tema señalan que en los procesos de duelo las personas con TEA pueden reaccionar de forma impredecible ante situaciones complicadas (p. e. generadoras de estrés o ansiedad) o hablar de temas que pueden ser percibidos por los demás como intrascendentes para ese momento (Gilrane-McGarry y Taggart, 2012). Ante esto, el instinto de las familias, al igual que ocurre con las personas con DI y con objeto de protegerlas, suele ser excluir a las personas con TEA de los rituales de duelo, ya que creen que no van a ser capaces de comprender la pérdida. Pese a esto, se ha demostrado que la inclusión y la participación en los rituales, así como los apoyos relacionados con la expresión y la superación de la pérdida, les ayudan a comprender mejor el proceso, además de enseñarles a procesar el dolor (Lawhon, 2004). Por lo tanto, debido a las escasas investigaciones realizadas sobre el duelo en personas con DI y discapacidades del desarrollo (más concretamente para el caso de las personas con TEA), nos planteamos la siguiente pregunta de investigación: ¿Cómo viven y experimentan estas personas el duelo?

Para tratar de dar respuesta a esta pregunta llevaremos a cabo una revisión sistemática de la literatura científica publicada en los últimos siete años. En esta, además de responder a la pregunta de investigación, nos planteamos también dar respuesta a los siguientes objetivos: (1) analizar si existen diferencias en el afrontamiento del duelo en función del género, edad y tipo de discapacidad de los participantes; (2) conocer los factores determinantes que provocan que las personas con DI y TEA afronten o asuman mejor o peor la pérdida; y (3) describir las estrategias más adecuadas para proporcionar apoyo a estas personas que se enfrentan a un proceso de duelo.

\section{Método}

\subsection{Materiales}

En primer lugar, para realizar un cribado de los artículos que se utilizarán en la revisión sistemática, se hizo una búsqueda en las siguientes bases de datos: CINAHL Complete, ERIC, MEDLINE, APA Psyarticles, PsycInfo y PSICODOC, todas ellas incluidas en la plataforma EBSCOhost. Dicha búsqueda fue complementada con otra manual realizada en las plataformas Proquest y ResearchGate. Todas las búsquedas se realizaron durante los meses de marzo y abril de 2020. 
FACTORES DETERMINANTES DEL DUELO EN PERSONAS

CON DISCAPACIDAD INTELECTUAL Y TEA: REVISIÓN SISTEMÁTICA

M. BÓVEDA HERMOSILLA Y N. FLORES ROBAINA

\subsection{Procedimiento}

Para la búsqueda se utilizaron como palabras clave los términos "bereavement or grief or loss" combinados con el operador booleano AND y las palabras clave representativas de las poblaciones objeto de análisis, esto es: "intellectual disabilities or learning disabilities"; “autism or asd or autism spectrum disorder”. Se determinó como período de búsqueda los últimos siete años, es decir, la producción científica referida a los años 2013 y 2020 (ambos inclusive). Así mismo, los criterios de inclusión que se establecieron para la selección de los artículos fueron los siguientes: (1) artículos académicos con revisión por pares (peer review); (2) publicados en idioma español o inglés; (3) artículos con metodología cuantitativa o cualitativa, de carácter descriptivo, experimental, transversal y/o longitudinal, estudios de caso, y con un tamaño muestral igual o superior a 1; (4) publicados durante el período de enero de 2013 a abril de 2020; (5) que versaran sobre la muerte, el proceso de duelo y su afrontamiento en la población de personas con DI y discapacidades del desarrollo (más concretamente TEA); (6) cuyos participantes fueran personas con DI o TEA como informantes directos; (7) que los artículos se encontraran disponibles para su lectura a texto completo.

En cuanto a los criterios de exclusión, se descartarían aquellos artículos que no cumplieran lo siguiente: (1) trabajos publicados en otros formatos diferentes al artículo académico (p. e. libros, capítulos de libro, tesis doctorales, actas de congreso, editoriales, respuesta a autores, artículos no académicos); (2) en otros idiomas diferentes del español o inglés; (3) que fuesen estudios de revisión teórica o narrativa, sistemática, o metaanálisis, o propuestas de programa de prevención o intervención sin tratamiento de datos; (4) cuya temática no tuviera como objetivo analizar los factores implicados en la muerte, el proceso de duelo y el afrontamiento en la población de personas con DI o discapacidades del desarrollo; (5) cuyas muestras no procedieran de personas con DI o TEA como informantes directos; (6) que no estuvieran disponibles a texto completo.

\subsection{Análisis de la información}

Tras la búsqueda inicial, el número total de publicaciones extraídas de la búsqueda en la plataforma EBSCOhost fue 577. Todas las bases de datos arrojaron resultados excepto APA Psyarticles. Más concretamente, los resultados obtenidos fueron: MEDLINE ( $\mathrm{n}=373$ ), APA PsycINFO $(\mathrm{n}=159)$, CINAHL Complete $(\mathrm{n}=32)$, ERIC $(n=12)$ y PSICODOC $(n=1)$. No se encontraron artículos duplicados. Por su parte, se identificaron otros ocho artículos tras la realización de una búsqueda manual adicional en las plataformas ProQuest $(n=2)$ y ResearchGate $(n=6)$. Resultando un total de 585 artículos, de los cuales 538 eran artículos académicos, siendo los 47 restantes otro tipo de documentos (p. e. tesis, libros e informes). Estos últimos fueron descartados, quedando un total de 538 artículos. Así, tras la lectura y análisis de los títulos y abstract se eliminaron 440 por no cumplir de entrada con los criterios 
de inclusión y más concretamente por tratar otras temáticas diferentes a la muerte y el duelo relacional. Tras este análisis, quedaron un total de 98 artículos, cuya lectura en profundidad nos llevó a descartar otros 84 artículos por no cumplir con los criterios de inclusión establecidos. Por lo que el total de artículos que conformarán la muestra de esta revisión sistemática es de 14 (véase Figura 1).

\section{Figura 1. Diagrama de flujo del proceso de búsqueda y selección de artículos}

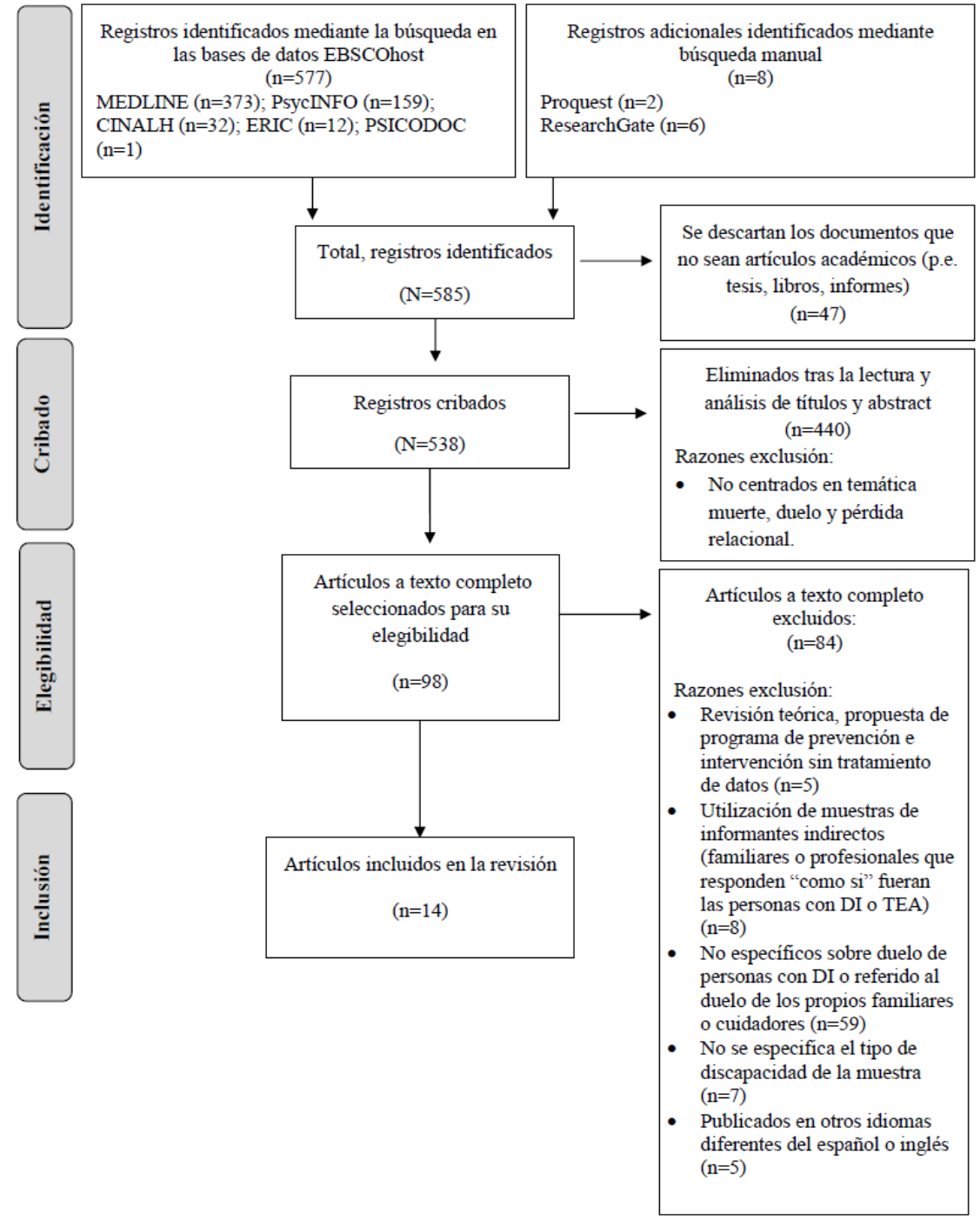

Ediciones Universidad de Salamanca / CC BY-NC-ND

Siglo Cero, vol. 52 (3), 2021, julio-septiembre, pp. 59-79

$$
-65-
$$




\section{Resultados}

\subsection{Características de los estudios revisados}

En la Tabla 1 se muestra un resumen de las características generales de los 14 artículos que conforman esta revisión. Respecto a los años de publicación, cabe observar cómo en los últimos cinco años ha incrementado el número de artículos académicos publicados sobre esta temática, pasando de un $35,71 \%$ de publicaciones realizadas durante los años 2013 a 2015 a un 64,28 \% durante el período de 2016 a 2019. En cuanto a los países de procedencia de los estudios, destacan Reino Unido (57,14 \% de las publicaciones), seguido de España (21,42 \%) y otros países como Estados Unidos, Australia o China $(7,14 \%)$. Respecto a las muestras de informantes, se puede observar cómo en todas las publicaciones la información procede de las personas con DI o TEA. No obstante, se ha de indicar que cuatro $(28,57 \%)$ trabajos se caracterizan por utilizar conjuntamente muestras de personas con DI y familiares o profesionales de atención directa a quienes también se ha evaluado en el mismo trabajo. En este caso, y por el interés que tiene para nuestro estudio, decidimos incluir estos trabajos en la revisión. En cuanto a la metodología utilizada en los trabajos, destacan los procedimientos cualitativos a través de los grupos focales y de discusión (42,85\%), frente a los trabajos de tipo cuantitativo $(28,57 \%)$ o frente al empleo de métodos mixtos $(28,57 \%)$.

\begin{tabular}{|l|l|l|l|l|l|}
\hline \multicolumn{5}{|c|}{ TABLA 1. Resumen y características generales de los artículos } \\
incluidos en la revisión
\end{tabular}


FACTORES DETERMINANTES DEL DUELO EN PERSONAS

CON DISCAPACIDAD INTELECTUAL Y TEA: REVISIÓN SISTEMÁTICA

M. BÓVEDA HERMOSILLA Y N. FLORES ROBAINA

\begin{tabular}{|c|c|c|c|c|c|}
\hline $\begin{array}{l}\text { Rodrí- } \\
\text { guez- } \\
\text { Herrero, } \\
\text { Izuzquiza } \\
\text { y De la } \\
\text { Herrán } \\
(2013)\end{array}$ & $\begin{array}{l}\text { Aplicación y } \\
\text { evaluación de } \\
\text { resultados de } \\
\text { un Programa } \\
\text { de Educación } \\
\text { para la Muer- } \\
\text { te para per- } \\
\text { sonas adultas } \\
\text { con DI }\end{array}$ & España & $\begin{array}{l}\text { Adultos con DI } \\
\text { ( } 9 \text { hombres; } 6 \\
\text { mujeres) }\end{array}$ & $\begin{array}{l}15 / 18 \text { a } 35 \text { años } \\
\text { / no se aporta la } \\
\text { media. }\end{array}$ & $\begin{array}{l}\text { Mixto. } \\
\text { Grupos de discusión. } \\
\text { Entrevista. Cuestio- } \\
\text { narios de elaboración } \\
\text { propia con medidas } \\
\text { pre-post }\end{array}$ \\
\hline $\begin{array}{l}\text { McRit- } \\
\text { chie, Mc- } \\
\text { Kenzie, } \\
\text { Quayle, } \\
\text { Harlin y } \\
\text { Neumann } \\
(2014)\end{array}$ & $\begin{array}{l}\text { Explorar la } \\
\text { experiencia } \\
\text { de duelo en } \\
\text { un grupo de } \\
\text { personas con } \\
\text { DI }\end{array}$ & Inglaterra & $\begin{array}{l}\text { Adultos con DI } \\
\text { ( } 8 \text { hombres; } 5 \\
\text { mujeres) }\end{array}$ & $\begin{array}{l}13 / 20 \text { a } 72 \text { años } \\
\text { / no se aporta la } \\
\text { media }\end{array}$ & $\begin{array}{l}\text { Cualitativo. } \\
\text { Análisis fenomeno- } \\
\text { lógico interpretativo } \\
\text { (IPA). Entrevista } \\
\text { semiestructurada }\end{array}$ \\
\hline $\begin{array}{l}\text { Rodrí- } \\
\text { guez- } \\
\text { Herrero, } \\
\text { Izuzquiza } \\
\text { y De la } \\
\text { Herrán } \\
(2015)\end{array}$ & $\begin{array}{l}\text { Explorar } \\
\text { el grado de } \\
\text { comprensión } \\
\text { de adultos } \\
\text { con DI sobre } \\
\text { las dimensio- } \\
\text { nes biológicas } \\
\text { de la muerte, } \\
\text { actitudes, } \\
\text { ideas y creen- } \\
\text { cias }\end{array}$ & España & $\begin{array}{l}\text { Adultos con DI } \\
\text { ( } 33 \text { hombres; } \\
42 \text { mujeres) }\end{array}$ & $\begin{array}{l}75 / 18 \text { a } 35 \text { años } \\
\text { / no se aporta la } \\
\text { media }\end{array}$ & $\begin{array}{l}\text { Mixto. } \\
\text { Cuestionarios estan- } \\
\text { darizados. Análisis } \\
\text { fenomenológico de } \\
\text { significados. Entre- } \\
\text { vista semiestructu- } \\
\text { rada }\end{array}$ \\
\hline $\begin{array}{l}\text { Stancliffe, } \\
\text { Wiese, } \\
\text { Read, } \\
\text { Jeltes y } \\
\text { Clayton } \\
(2016)\end{array}$ & $\begin{array}{l}\text { Comparar } \\
\text { el grado de } \\
\text { conocimien- } \\
\text { to sobre la } \\
\text { muerte, la } \\
\text { viabilidad de } \\
\text { planificar el } \\
\text { final de la vida } \\
\text { y el miedo a la } \\
\text { muerte, entre } \\
\text { personas con } \\
\text { DI y sin ella }\end{array}$ & Australia & $\begin{array}{l}\text { Adultos con DI } \\
\text { (25 hombres; } \\
14 \text { mujeres) } \\
\text { Profesionales } \\
\text { de atención } \\
\text { a personas } \\
\text { con DI (13 } \\
\text { hombres; } 27 \\
\text { mujeres) }\end{array}$ & $\begin{array}{l}39 \text { adultos con DI / } \\
\text { no se indica rango } \\
\text { edad / } M=48,62 \\
\text { años } \\
40 \text { profesionales } \\
\text { sector DI / no se } \\
\text { indica rango edad / } \\
M=41,60 \text { años. }\end{array}$ & $\begin{array}{l}\text { Cuantitativo. } \\
\text { Cuestionarios estan- } \\
\text { darizados }\end{array}$ \\
\hline $\begin{array}{l}\text { Young y } \\
\text { Garrard } \\
(2016)\end{array}$ & $\begin{array}{l}\text { Explorar la } \\
\text { vivencia del } \\
\text { duelo en una } \\
\text { mujer con DI } \\
\text { severa }\end{array}$ & Escocia & $\begin{array}{l}\text { Adulta con DI } \\
\text { (1 mujer) }\end{array}$ & $1 / 26$ años & $\begin{array}{l}\text { Cualitativo. } \\
\text { Estudio de caso } \\
\text { único. Metodología } \\
\text { observacional. Análi- } \\
\text { sis del discurso }\end{array}$ \\
\hline
\end{tabular}

Ediciones Universidad de Salamanca / CC BY-NC-ND

Siglo Cero, vol. 52 (3), 2021, julio-septiembre, pp. 59-79

$$
-67-
$$


FACTORES DETERMINANTES DEL DUELO EN PERSONAS

CON DISCAPACIDAD INTELECTUAL Y TEA: REVISIÓN SISTEMÁTICA

M. BÓVEDA HERMOSILLA Y N. FLORES ROBAINA

\begin{tabular}{|c|c|c|c|c|c|}
\hline $\begin{array}{l}\text { Chow, } \\
\text { McEvoy, } \\
\text { Chan, } \\
\text { Borschel, } \\
\text { Yuen y } \\
\text { Lo (2017) }\end{array}$ & $\begin{array}{l}\text { Investigar en } \\
\text { qué medida } \\
\text { las perso- } \\
\text { nas con DI } \\
\text { comprenden } \\
\text { los conceptos } \\
\text { de muerte y } \\
\text { duelo }\end{array}$ & China & $\begin{array}{l}\text { Adultos DI } \\
\text { ( } 65 \text { hombres; } \\
45 \text { mujeres) }\end{array}$ & $\begin{array}{l}111 / \text { no se indica } \\
\text { rango edad / } \\
M=39.81 \text { años }\end{array}$ & $\begin{array}{l}\text { Cuantitativo. } \\
\text { Cuestionarios estan- } \\
\text { darizados }\end{array}$ \\
\hline $\begin{array}{l}\text { Irwin, } \\
\text { O'Malley, } \\
\text { Neelo- } \\
\text { four y } \\
\text { Guerin } \\
\text { (2017) }\end{array}$ & \begin{tabular}{|l|} 
Explorar los \\
apoyos que, \\
desde la psi- \\
cología clíni- \\
ca, se ofrecen \\
a los adultos \\
con DI en \\
duelo desde la \\
perspectiva de \\
las personas \\
con DI en \\
duelo y los \\
profesionales \\
de apoyo
\end{tabular} & Irlanda & $\begin{array}{l}\text { Adultos con DI } \\
\text { ( } 3 \text { hombres; } 3 \\
\text { mujeres) } \\
\text { Profesionales } \\
\text { de atención } \\
\text { directa } \\
\text { (2 hombres; } 4 \\
\text { mujeres) }\end{array}$ & $\begin{array}{l}6 \text { personas con DI / } \\
22.25 \text { a } 43.25 \text { / M = } \\
39.5 \text { años } \\
6 \text { profesionales de } \\
\text { atención a personas } \\
\text { con DI / no se } \\
\text { indica el rango de } \\
\text { edad / no se aporta } \\
\text { media }\end{array}$ & $\begin{array}{l}\text { Mixto. } \\
\text { Análisis de casos. } \\
\text { Entrevista y cuestio- } \\
\text { narios estandarizados }\end{array}$ \\
\hline $\begin{array}{l}\text { McEvoy, } \\
\text { Treacy y } \\
\text { Quigley } \\
\text { (2017) }\end{array}$ & $\begin{array}{l}\text { Conocer la } \\
\text { relación entre } \\
\text { el conoci- } \\
\text { miento de los } \\
\text { componentes } \\
\text { biológicos } \\
\text { asociados con } \\
\text { el proceso } \\
\text { de morir y la } \\
\text { comprensión } \\
\text { del concepto } \\
\text { de muerte en } \\
\text { personas con } \\
\text { DI } \\
\end{array}$ & Irlanda & $\begin{array}{l}\text { Adultos con DI } \\
\text { (14 hombres; } \\
16 \text { mujeres) }\end{array}$ & $\begin{array}{l}30 / 19 \text { a } 74 \text { años / } \\
M=38.99 \text { años }\end{array}$ & $\begin{array}{l}\text { Cuantitativo. } \\
\text { Cuestionarios } \\
\text { estandarizados y } \\
\text { entrevista }\end{array}$ \\
\hline $\begin{array}{l}\text { Zakreski } \\
\text { (2017) }\end{array}$ & $\begin{array}{l}\text { Realizar } \\
\text { intervención } \\
\text { psicológica } \\
\text { sobre el duelo } \\
\text { con objeto } \\
\text { de mejorar la } \\
\text { comprensión } \\
\text { y manejo de } \\
\text { los conceptos } \\
\text { de muerte y } \\
\text { pérdida }\end{array}$ & EE. UU. & $\begin{array}{l}\text { Adolescente } \\
\text { con TEA } \\
(1 \text { mujer })\end{array}$ & $1 / 15$ años & $\begin{array}{l}\text { Cualitativo. } \\
\text { Estudio de caso } \\
\text { único. Entrevista }\end{array}$ \\
\hline
\end{tabular}

Ediciones Universidad de Salamanca / CC BY-NC-ND

Siglo Cero, vol. 52 (3), 2021, julio-septiembre, pp. 59-79

$$
-68-
$$


FACTORES DETERMINANTES DEL DUELO EN PERSONAS CON DISCAPACIDAD INTELECTUAL Y TEA: REVISIÓN SISTEMÁTICA M. BÓVEDA HERMOSILLA Y N. FLORES ROBAINA

\begin{tabular}{|c|c|c|c|c|c|}
\hline $\begin{array}{l}\text { Domingo, } \\
\text { Cuesta y } \\
\text { Sánchez } \\
(2018)\end{array}$ & $\begin{array}{l}\text { Conocer } \\
\text { cómo los jó- } \\
\text { venes con DI } \\
\text { y su entorno } \\
\text { viven los pro- } \\
\text { cesos de duelo } \\
\text { causados } \\
\text { por pérdidas } \\
\text { relacionales }\end{array}$ & España & $\begin{array}{l}\text { Adultos con } \\
\text { DI. } \\
\text { (no se informa } \\
\text { sobre el sexo) } \\
\text { Familiares y } \\
\text { profesionales } \\
\text { (no se informa } \\
\text { sobre el sexo) }\end{array}$ & $\begin{array}{l}12 \text { adultos con DI / } \\
16 \text { a } 25 \text { años / no se } \\
\text { aporta la media } \\
9 \text { familiares de } \\
\text { personas con DI / } \\
\text { no se aporta la edad } \\
\text { ni la media. } \\
8 \text { profesionales de } \\
\text { atención a personas } \\
\text { con DI / no se } \\
\text { aporta la edad ni la } \\
\text { media }\end{array}$ & $\begin{array}{l}\text { Cualitativo. } \\
\text { Grupos de discusión }\end{array}$ \\
\hline $\begin{array}{l}\text { Thorp, } \\
\text { Stedmon } \\
\text { y Lloyd } \\
(2018)\end{array}$ & $\begin{array}{l}\text { Explorar la } \\
\text { experiencia } \\
\text { de duelo de } \\
\text { personas con } \\
\text { DI }\end{array}$ & Inglaterra & $\begin{array}{l}\text { Adultos con DI } \\
\text { ( } 2 \text { hombres; } 2 \\
\text { mujeres) }\end{array}$ & $\begin{array}{l}4 \text { adultos con DI / } \\
32 \text { a } 40 \text { años / } M= \\
33 \text { años }\end{array}$ & $\begin{array}{l}\text { Cualitativo. } \\
\text { Análisis fenomeno- } \\
\text { lógico interpretativo } \\
\text { (IPA). Entrevistas } \\
\text { semiestructuradas }\end{array}$ \\
\hline $\begin{array}{l}\text { O’Keffe, } \\
\text { Guerin, } \\
\text { McEvoy, } \\
\text { Lockhart } \\
\text { y Dodd } \\
\text { (2019) }\end{array}$ & $\begin{array}{l}\text { Desarrollar } \\
\text { un instru- } \\
\text { mento de } \\
\text { autoinforme } \\
\text { para el duelo } \\
\text { complicado } \\
\text { en personas } \\
\text { con DI, a } \\
\text { partir de sus } \\
\text { concepciones } \\
\text { y experien- } \\
\text { cias sobre la } \\
\text { muerte y el } \\
\text { duelo }\end{array}$ & Irlanda & $\begin{array}{l}\text { Adultos con DI } \\
\text { ( } 5 \text { hombres; } 5 \\
\text { mujeres) }\end{array}$ & $\begin{array}{l}16 \text { personas con } \\
\text { DI / no se aporta } \\
\text { información sobre } \\
\text { la edad ni la media }\end{array}$ & $\begin{array}{l}\text { Mixto. } \\
\text { Cuestionario estan- } \\
\text { darizado. } \\
\text { Grupos focales }\end{array}$ \\
\hline
\end{tabular}

La muestra de participantes analizada globalmente y pertenecientes a los 14 artículos que componen esta revisión asciende a un total de 342 personas con DI y TEA, de las cuales $174(50,88 \%)$ fueron hombres frente a $149(43,57 \%)$ mujeres, cuyas edades oscilaron entre los 15 y los 93 años. En siete de los trabajos (50\%), no se aportó información sobre la edad media de los participantes. Sin embargo, en los otros siete artículos en los que sí se aportó este dato, encontramos que la media de edad de los participantes osciló entre los 33 y los 48,62 años. En cuanto al diagnóstico principal de los participantes, encontramos que un $92,86 \%$ tienen discapacidad intelectual frente al diagnóstico de TEA (7,14 \%). Respecto al grado de severidad en la discapacidad, más de la mitad de los participantes con DI presentaron niveles leves a moderados $(57,14 \%)$ frente a quienes tenían niveles severos (28,57\%). Además, en tres de los 14 estudios no se indicó el grado de discapacidad de los participantes (14,29\%). 
FACTORES DETERMINANTES DEL DUELO EN PERSONAS

CON DISCAPACIDAD INTELECTUAL Y TEA: REVISIÓN SISTEMÁTICA

M. BÓVEDA HERMOSILLA Y N. FLORES ROBAINA

\subsection{Afrontamiento del duelo y factores determinantes}

Con objeto de responder al primer objetivo, consistente en analizar si existirán diferencias en el afrontamiento del duelo en función del género, la edad y el tipo de discapacidad de los participantes, tras la revisión de los estudios, se puede afirmar que no se encontraron diferencias en el afrontamiento en función del género (Borsay et al., 2013; Chow et al., 2017; Forrester-Jones, 2013; Irwin et al., 2017; RodríguezHerrero et al., 2015; Stancliffe et al., 2016). En cuanto a si la edad influirá en el afrontamiento del duelo, los resultados obtenidos son contradictorios. Así, por ejemplo, Rodríguez-Herrero et al. (2015) sí encuentran relación entre la edad de los participantes y su comprensión sobre la muerte, mientras que otros autores (McRitchie et al., 2014) aluden a que la edad en sí no provoca un mejor o peor afrontamiento del duelo, sino que son las experiencias previas vividas por la persona. Por último, otra de las variables que parece influir en la comprensión de la muerte y el afrontamiento del duelo es el tipo de discapacidad y su grado. En este sentido, en el trabajo de McRitchie et al. (2014) se constata que los participantes tienen una comprensión aceptable de la muerte y sus consecuencias, pero muestran dificultades a la hora de expresar o comunicar lo que sienten, ya que la comunicación verbal y la expresión emocional limitada parecen dificultar su interacción con los demás. En el caso de las personas con TEA, también presentan dificultades para expresarse emocionalmente y en algunos casos problemas en el procesamiento, la concentración y la comprensión de la información (Zakreski, 2017). Con respecto a las personas con DI, la mayoría de los estudios revisados (Chow et al., 2017; Irwin et al., 2017; McEvoy et al., 2017; O’Keffe et al., 2018; Rodríguez-Herrero et al., 2013, 2015) concluyen que las personas con DI de tipo leve comprenden mejor el concepto de la muerte que aquellas con niveles de discapacidad moderados o severos.

En relación con el segundo objetivo planteado consistente en conocer los factores que determinan que las personas con DI y discapacidades del desarrollo afronten y/o asuman mejor o peor la pérdida, tras el análisis de los trabajos encontramos dos grandes categorías bajo las que se pueden agrupar dichos factores: los factores "internos a la persona" y los "externos". En la Tabla 2 presentamos dichos factores y el porcentaje en que son tratados en las publicaciones revisadas. Así, es posible indicar cómo el conocimiento y la comprensión de los conceptos asociados a la muerte (irreversibilidad, universalidad), así como sus componentes biológicos, es el factor interno de mayor relevancia para las personas con DI y del desarrollo. Sobre este se concluye que, si bien las personas con DI entienden el concepto de muerte, parecen tener un conocimiento bastante limitado respecto a los componentes relacionados con esta. Dicha incomprensión se acentúa, además, conforme aumenta el grado de severidad en la discapacidad y provoca dificultades para afrontar de una manera normalizada la pérdida (Borsay et al., 2013; Chow et al., 2017; Domingo et al., 2018; Irwin et al., 2017; McEvoy et al., 2017; McRitchie et al., 2014; Rodríguez-Herrero et al., 2013, 2015; Stancliffe et al., 2016). Por otro lado, el factor externo de mayor importancia y más referenciado es la participación e inclusión de la persona con DI en los rituales sobre el duelo, encontrándose en la mitad de los trabajos revisados (Borsay et al., 
2013; Domingo et al., 2018; Forrester-Jones, 2013; McRitchie et al., 2014; Rodríguez et al., 2013, 2015; Thorp et al., 2018). Otros factores como haber tenido experiencias previas de pérdidas (McRitchie et al., 2014; Stancliffe et al., 2016), el grado de vinculación con la persona fallecida (Domingo et al., 2018; Young y Garrard, 2016; Zakreski, 2017) o presentar otras pérdidas adicionales secundarias a la pérdida principal (Forrester-Jones, 2013; Thorp et al., 2018) también afectan a la experiencia del afrontamiento, aunque en menor medida.

TABLA 2. Factores determinantes en el afrontamiento del duelo de personas con DI y discapacidades del desarrollo

\begin{tabular}{|c|c|c|}
\hline Factores internos & $\begin{array}{l}\text { Porcentaje de trabajos que } \\
\text { analizan el factor } \\
(\%)\end{array}$ & Conclusiones \\
\hline $\begin{array}{l}\text { Comprensión de los } \\
\text { conceptos asociados a la } \\
\text { muerte }\end{array}$ & $64 \%$ & $\begin{array}{l}\text { Un mayor conocimiento de los } \\
\text { conceptos asociados a la muerte } \\
\text { contribuye a un mejor afronta- } \\
\text { miento del duelo }\end{array}$ \\
\hline Experiencia previa & $28 \%$ & $\begin{array}{l}\text { Las experiencias previas de pér- } \\
\text { dida implican una adquisición } \\
\text { del concepto de muerte y un } \\
\text { mejor afrontamiento del duelo }\end{array}$ \\
\hline Grado de vínculo & $21 \%$ & $\begin{array}{l}\text { Un mayor vínculo con la per- } \\
\text { sona fallecida provoca mayores } \\
\text { dificultades para afrontar el } \\
\text { duelo ya que la sensación de } \\
\text { pérdida es mayor }\end{array}$ \\
\hline Factores externos & $\begin{array}{c}\text { Porcentaje de trabajos que } \\
\text { analizan el factor } \\
(\%)\end{array}$ & Conclusiones \\
\hline $\begin{array}{l}\text { Inclusión/exclusión en } \\
\text { rituales }\end{array}$ & $50 \%$ & $\begin{array}{l}\text { La inclusión de la persona con } \\
\text { DI en los rituales de la muerte } \\
\text { genera un mejor afrontamiento } \\
\text { del duelo }\end{array}$ \\
\hline $\begin{array}{l}\text { Otras pérdidas secundarias } \\
\text { o adicionales }\end{array}$ & $14 \%$ & $\begin{array}{l}\text { Las pérdidas secundarias o } \\
\text { adicionales como la pérdida del } \\
\text { cuidador principal o un cambio } \\
\text { de residencia pueden agravar el } \\
\text { proceso de duelo }\end{array}$ \\
\hline Residencia & $14 \%$ & $\begin{array}{l}\text { Los entornos residenciales } \\
\text { ofrecen unas oportunidades más } \\
\text { limitadas para afrontar el duelo } \\
\text { y recibir apoyo }\end{array}$ \\
\hline
\end{tabular}




\subsection{Estrategias de apoyo frente al duelo}

Respecto al tercer objetivo consistente en describir las estrategias más adecuadas para proporcionar apoyo a las personas con DI y discapacidades del desarrollo que se enfrentan al proceso de duelo, hemos hallado diferentes estrategias que se pueden dividir en tres bloques, en función del momento en el que se deberían trabajar con el doliente. Así, cabría hablar de una serie de estrategias a ofrecer en momentos "previos" a la pérdida del ser querido. En segundo lugar, se encontrarían aquellas que podrían aplicarse una vez ha tenido lugar el fallecimiento. Y, por último, destacarían las estrategias que podrían utilizarse tanto antes como después del fallecimiento. En la Tabla 3 presentamos los resultados obtenidos respecto a las diferentes estrategias. Así, la estrategia reconocida como más eficaz por las personas con DI participantes en los trabajos revisados fue la participación en los rituales de muerte, tal y como se constata en el estudio de Forrester-Jones (2013), donde las propias personas con DI indicaron que el no haber sido invitadas al funeral de la persona significativa les privaba de la oportunidad para compartir su dolor con otros miembros. Por su parte, brindar información sobre el estado de salud de la persona querida y preparar anticipadamente a la persona con discapacidad sobre el fallecimiento de su familiar es otra de las estrategias que facilita la adaptación y el afrontamiento en el doliente (Irwin et al., 2017; McEvoy et al., 2017). Otra de las estrategias identificadas como relevantes fue la mejora de la comunicación y la expresión emocional. En este caso, ayudar a las personas con DI y TEA a expresar el dolor les protege frente al desarrollo de un duelo complicado (Thorp et al., 2018; Zakreski, 2017).

\begin{tabular}{|c|c|c|}
\hline \multicolumn{3}{|c|}{$\begin{array}{l}\text { TABLA 3. Estrategias eficaces en el afrontamiento del duelo } \\
\text { en personas con DI y discapacidades del desarrollo }\end{array}$} \\
\hline Previas a la muerte & $\begin{array}{l}\text { Porcentaje de trabajos que } \\
\text { refieren la estrategia } \\
(\%)\end{array}$ & Conclusiones \\
\hline Preparación para la muerte & $43 \%$ & $\begin{array}{l}\text { Proporcionar información sobre } \\
\text { el estado de salud y trabajar } \\
\text { la muerte anticipada mejora el } \\
\text { afrontamiento en la persona con } \\
\text { DI }\end{array}$ \\
\hline Posteriores a la muerte & $\begin{array}{c}\text { Porcentaje de trabajos que } \\
\text { refieren la estrategia } \\
(\%)\end{array}$ & Conclusiones \\
\hline Inclusión en rituales & $54 \%$ & $\begin{array}{l}\text { La inclusión y participación } \\
\text { activa en los rituales genera sa- } \\
\text { tisfacción y protege de un duelo } \\
\text { complicado }\end{array}$ \\
\hline
\end{tabular}


FACTORES DETERMINANTES DEL DUELO EN PERSONAS

CON DISCAPACIDAD INTELECTUAL Y TEA: REVISIÓN SISTEMÁTICA

M. BÓVEDA HERMOSILLA Y N. FLORES ROBAINA

\begin{tabular}{|c|c|l|}
\hline $\begin{array}{l}\text { Recepción de apoyo fami- } \\
\text { liar y profesional }\end{array}$ & $28 \%$ & $\begin{array}{l}\text { Que las personas con DI } \\
\text { cuenten con apoyo familiar y } \\
\text { profesional adecuado a sus nece- } \\
\text { sidades mejora el afrontamiento }\end{array}$ \\
\hline $\begin{array}{c}\text { A utilizar en ambos mo- } \\
\text { mentos }\end{array}$ & $\begin{array}{c}\text { Porcentaje de trabajos que } \\
\text { refieren la estrategia } \\
(\%)\end{array}$ & Conclusiones \\
\hline $\begin{array}{c}\text { Mejorar la comunicación y } \\
\text { expresión de sentimientos }\end{array}$ & $21 \%$ & $\begin{array}{l}\text { Expresar sentimientos y comu- } \\
\text { nicarlos al entorno facilita la } \\
\text { adaptación al duelo }\end{array}$ \\
\hline
\end{tabular}

\section{Discusión}

Esta revisión sistemática tenía como objetivo explorar el proceso de duelo de las personas con DI y discapacidades del desarrollo, así como conocer los factores que influyen en él y las estrategias para un mejor afrontamiento. Los resultados indicaron que la variable sexo no influyó en el afrontamiento del duelo, pero sí lo hizo el tipo de discapacidad (Rodríguez et al., 2015; McRitchie et al., 2014). Estos resultados coinciden con los encontrados en otros trabajos donde profesionales de atención directa evalúan el duelo de personas con DI (Alcedo et al., 2018; Muñiz et al., 2017). Por su parte, algunos de los factores más importantes en el afrontamiento del duelo son la comprensión de los conceptos de la muerte, la inclusión o exclusión de los rituales o la experiencia previa. En diferentes trabajos se constata que estos factores están muy relacionados entre sí, ya que, si una persona con DI o TEA comprende lo que supone la muerte y ha vivido alguna experiencia de duelo a lo largo de su vida, es probable que ante el fallecimiento de una persona significativa sepa reaccionar de manera más adaptativa que una persona que no ha vivido estas experiencias (Rodríguez et al., 2015; Zakreski, 2017). Otros autores como Delorme (1999), sin embargo, han comprobado que un fuerte vínculo con la persona fallecida podría suponer un suceso traumático para la persona con discapacidad. Idea defendida por otros autores y que quizás pudiera contribuir a explicar parcialmente las razones de excluir de los rituales a estas personas (Dodd et al., 2008; McAdams y Stoughb, 2018). Frente a esto, no han sido pocos los estudios de esta revisión que han demostrado que la inclusión de las personas con discapacidad intelectual en los rituales produce en ellas un sentimiento de satisfacción, un aumento de la autoestima y confianza en sí mismos, además de una mejora de su inclusión social y calidad de vida (Domingo et al., 2018; Forrester-Jones, 2013; Stancliffe et al., 2016; Thorp et al., 2018; Zakreski, 2017). Dichos resultados coinciden ampliamente con los modelos teóricos respecto al proceso de afrontamiento del duelo en la población en general y también refrendan los hallazgos encontrados en otros estudios de revisión realizados en la población de personas con discapacidad intelectual (Cristóbal et al., 2018; Worden, 2013). En ellos se constata cómo una de las primeras tareas a realizar cuando atravesamos un proceso de duelo por la pérdida de un ser querido consiste en aceptar la realidad de la pérdida y su irreversibilidad. Par- 
ticipar en los rituales relacionados con la muerte, como por ejemplo asistir al entierro o funeral del fallecido, ha demostrado ser una gran ayuda que contribuye en la validación de la muerte y en el afrontamiento más saludable del proceso del duelo posterior. Por lo que permitir a las personas con DI y del desarrollo participar de estos eventos se considera un paso importante a la hora de favorecer el afrontamiento y proteger de un posible duelo complicado. No obstante, resultará necesario incrementar la investigación sobre este tema en aras de aportar resultados sobre el impacto que pudiera tener la participación en este tipo de rituales para aquellas personas con DI y TEA con grandes necesidades de apoyo. No cabe duda de que conocer y determinar qué tipo de estrategias serían las más apropiadas y útiles en estos casos constituye una cuestión pendiente aún de clarificar y un reto a acometer en futuros estudios sobre el tema.

También en esta revisión hemos encontrado evidencias a favor de que las personas con DI viven y experimentan el duelo de diferentes maneras. La forma en que muestran su dolor dependerá, en gran medida, de su capacidad para expresarse y comunicar sus sentimientos (Chow et al., 2017; McRitchie et al., 2014; Young y Garrard, 2016). Al respecto, estudios realizados con familiares y profesionales de atención directa admiten que en algunas ocasiones se interpretan erróneamente las expresiones del duelo de las personas con DI, atribuyéndose a las características propias de la discapacidad y no al dolor que experimentan como consecuencia de la pérdida de un ser querido (Alcedo et al., 2018; Brickell y Munir, 2008; McAdams y Stoughb, 2018). Estos resultados refrendan el argumento de Hume et al. (2016), quienes defienden que los cuidadores pueden no reconocer el aumento de estos comportamientos desafiantes como expresión del dolor y estas respuestas pueden ser minimizadas y malinterpretadas. Por tanto, proporcionar espacios donde las personas con DI se puedan comunicar y expresar sus emociones, así como utilizar herramientas de apoyo y adaptadas al tipo de discapacidad (p. e., ayudas visuales, cajas de memoria multisensoriales, utilización de fotografías o vídeos, etc.), contribuirán a la mejora del bienestar emocional y protegerán de un posible duelo complicado (Domingo et al., 2018; Thorp et al., 2018; Young y Garrard, 2016). De hecho, los hallazgos obtenidos corroboran la importancia de la utilización adicional de un lenguaje sencillo y directo, evitando los eufemismos y los conceptos abstractos, especialmente en el trabajo sobre el duelo con personas que presenten trastornos del espectro del autismo (Koehler, 2016; Zakreski, 2017).

Otro hallazgo importante de este trabajo ha sido confirmar que lo que más parece ayudar a las personas con DI a sobrellevar la pérdida es simplemente hablar sobre ello. En este sentido los participantes del estudio de McRitchie et al. (2014) destacaron como muy positiva la oportunidad de compartir el dolor y dialogar con personas que estaban pasando por su misma situación. Resultados que coinciden con los planteamientos ofrecidos por Carroll (2013). Permitir estos espacios a las personas con discapacidad mediante la puesta en marcha de intervenciones grupales de apoyo al duelo podría contribuir a una mejor elaboración de los procesos de duelo y servir de protección ante el desarrollo de un posible duelo patológico.

En contraposición a lo concluido anteriormente sobre la escasez de investigaciones en las que las personas con discapacidad fueran partícipes de los rituales de muer- 
te, estudios como los realizados por McRitchie et al. (2014), Rodríguez-Herrero et al. (2015) o Thorp et al. (2018) demuestran que en los últimos años cada vez más personas son incluidas en este tipo de rituales y es algo muy positivo para ellas. Cabe decir que, aunque este hecho constituye un gran avance, las investigaciones existentes sobre esta inclusión en personas con TEA son aún muy escasas. Esto reclama la necesidad de prestar especial atención al duelo de este colectivo.

Finalmente, no debemos olvidarnos de prestar atención a quienes se encargan de apoyar a las personas en duelo, ya que se ha comprobado que no cuentan con la formación adecuada para realizar estas tareas (Lord et al., 2017; McCLean y Guerin, 2019; Tuffrey-Wijne y Rose, 2017). Si este apoyo no se realiza adecuadamente, puede convertir el duelo de la persona con discapacidad en patológico y provocar problemas de salud mental (Zakreski, 2017). Por tanto, la formación de los profesionales en materia de duelo constituye también otro reto de futuro en este ámbito.

En conclusión, los hallazgos de esta revisión sistemática nos han llevado a identificar varios aspectos a tener en cuenta para la práctica. Uno de ellos es la constatación de la importancia que tiene educar en la pedagogía de la muerte a las personas con DI y del desarrollo, de forma adecuada a sus capacidades, desde una planificación de apoyos centrados en la persona. Así se evitará que se excluya erróneamente a estas personas de los rituales de muerte por pensar que no son capaces de comprenderlos y se les dará la oportunidad de que participen activamente para tener un mejor afrontamiento del duelo. Por otro lado, la escasa información sobre el tema de la muerte y el duelo en personas con TEA provoca que las conclusiones a las que hemos llegado sean más imprecisas en comparación con las investigaciones existentes sobre DI que, siendo también insuficientes, son más numerosas. Es necesario, por tanto, incrementar la investigación en el colectivo con TEA, de cara a conocer de primera mano cómo viven y experimentan los procesos de duelo, así como diseñar y poner en marcha estrategias de intervención ajustadas a sus necesidades y con los apoyos adecuados ( $\mathrm{p}$. e., utilización de pictogramas, agendas visuales, etc.).

Finalmente, conviene indicar que esta revisión sistemática ha tenido también varias limitaciones. En primer lugar, hay que destacar que los escasos artículos encontrados para esta revisión provocan que la muestra de participantes sobre la que se ha obtenido la información sea relativamente pequeña y, por tanto, los resultados no puedan generalizarse. Otra limitación tendría que ver con la disponibilidad de los artículos a los que hemos podido acceder en este trabajo. En este sentido, probablemente existan otros artículos y trabajos científicos que hayan podido quedar fuera de esta revisión debido a las dificultades encontradas para el acceso a texto completo. Así mismo, también pudieran existir otros trabajos de investigación procedentes de la literatura gris que aporten información relevante y no hayamos tenido en cuenta en esta revisión. Futuros trabajos podrán realizarse en aras de ampliar los procesos de búsqueda y superar así esta debilidad.

Así mismo, la escasa participación de las personas con DI y discapacidades del desarrollo como informantes directos de sus procesos de duelo constituye otra limitación a superar en futuras investigaciones. Finalmente, la escasez de estudios longitudinales y la disparidad y falta de uniformidad respecto a los instrumentos de 
FACTORES DETERMINANTES DEL DUELO EN PERSONAS

CON DISCAPACIDAD INTELECTUAL Y TEA: REVISIÓN SISTEMÁTICA

M. BÓVEDA HERMOSILLA Y N. FLORES ROBAINA

evaluación empleados es otra de las principales limitaciones de las que adolece este campo de estudio, tal y como también se ha reflejado en otras revisiones sobre el tema (Cristóbal et al., 2017; Fernández-Ávalos et al., 2017).

Pese a ello, consideramos que los resultados derivados de esta revisión son relevantes, aunque será necesario seguir aportando información sobre el estado de la cuestión de este campo de investigación en aras de incrementar la investigación y desarrollar prácticas basadas en la evidencia, además de visibilizar las necesidades de un colectivo al que se le ha venido privando de un derecho fundamental como es el derecho a poder despedirse y elaborar el duelo por sus seres queridos.

\section{Referencias bibliográficas}

\section{* Artículos incluidos en la revisión sistemática}

Alcedo, M. Á., Cristóbal, L., Gómez, L. E. y Arias, V. B. (2018). Evaluating the characteristics of the grieving process in people with intellectual disability. Journal of Applied Research in Intellectual Disabilities, 31(6), 999-1007. https://doi.org/10.1111/jar.12454

Barreto, P., De la Torre, O. y Pérez, M. (2012). Detección del duelo complicado. Psicooncología, 9(2-3), 355-368. https://doi.org/10.5209/rev_Psic.2013.v9.n2-3.40902

Bergman, E. J., Haley, W. E. y Small, B. J. (2011). Who uses bereavement services? An examination of service use by bereaved dementia caregivers. Aging and Mental Health, 15, 531-540. https://doi.org/10.1080/13607863.2010.543661

Blackman, N. (2008). The development of an assessment tool for the bereavement needs of people with learning disabilities. British Journal of Learning Disabilities, 36(3), 165-170. https://doi.org/10.1111/j.1468-3156.2008.00514.x

*Borsay, C., Halsey, M. y Critoph, A. (2013). Planning, facilitating and evaluating a bereavement group for adults with learning disabilities living in the community. British Journal of Learning Disabilities, 41(4), 266-272. https://doi.org/10.1111/j.1468-3156.2012.00751.x

BrestafF, K. (1984). Reactions to death: can the mentally handicapped grieve? Some experience of those who did. Teaching and Training, 22(1), 10-16.

Brickell, C. y Munir, K. (2008). El duelo y sus complicaciones en las personas con discapacidad intelectual. Revista Síndrome de Down, 25(2), 68-76.

Carroll, D. W. (2013). Families of children with developmental disablities: understanding stress and opportunities for growth. American Psychological Association. https://doi. org/10.1037/14192-000

Cathcart, F. (1995). Death and people with learning disabilities: interventions to support clients and carers. British Journal of Clinical Psychology, 34(2), 165-175. https://doi. org/10.1111/j.2044-8260.1995.tb01452.x

*Chow, A. Y. M., Mcevoy, J., Chan, I. K. N., Borschel, M., Yuen, J. H. L. y Lo, J. Y. M. (2017). Do men and women with intellectual disabilities understand death? Journal of Intellectual Disability Research, 61(12), 1130-1139. https://doi.org/10.1111/jir.12431

Cristóbal, L., Alcedo, M. A. y Gómez, L. E. (2017). Duelo en discapacidad intelectual: los avances de una década. Revista Española de Discapacidad, 5(2), 53-72. https://doi. org/10.5569/2340-5104.05.02.03 
FACTORES DETERMINANTES DEL DUELO EN PERSONAS

CON DISCAPACIDAD INTELECTUAL Y TEA: REVISIÓN SISTEMÁTICA

M. BÓVEDA HERMOSILLA Y N. FLORES ROBAINA

Delorme, M. (1999). Ageing and people with developmental disabilities. En I. Brown y M. Percy (Eds.), Developmental disabilities in Ontario (pp. 189-195). Front Porch Publishing.

Dodd, P., Dowling, S. y Hollins, S. (2005). A review of the emotional, psychiatric and behavioural responses to bereavement in people with intellectual disabilities. Journal of Intellectual Disability Research, 49(7), 537-543. https://doi.org/10.1111/j.1365-2788.2005.00702.x

Dodd, P., Guerin, S., Mcevoy, J., Buckley, S., Tyrrell, J. y Hillery, J. (2008). A study of complicated grief symptoms in people with intellectual disabilities. Journal of Intellectual Disability Research, 52(5), 415-425. https://doi.org/ 10.1111/j.1365-2788.200801043.x

*Domingo, E., Cuesta, J. L. y SÁnchez, S. (2018). Cómo afrontar el duelo en las personas con discapacidad. Una aproximación al problema. Siglo Cero, 49(4), 51-68. https://doi. org/10.14201/scero20184945168

Dowling, S., Hubert, J., White, S. y Hollins, S. (2006). Bereaved adults with intellectual disabilities: a combined randomized controlled trial and qualitative study of two communitybased interventions. Journal of Intellectual Disability Research, 50(4), 277-287. https://doi. org/10.1111/j.1365-2788.2005.00759.x

Fernández-Ávalos, M., Fernández-Alcántara, M., Cruz-Quintana, F. y Pérez-Marfil, M. N. (2017). Assessment of the grieving processes in people with intellectual disabilities: a systematic review. Estudios de Psicología, 1-9. https://doi.org/10.1080/02109395.2017.13 28846

*Forrester-Jones, R. (2013). The road barely taken: funerals, and people with intellectual disabilities. Journal of Applied Research in Intellectual Disabilities, 26(3), 243-256. https:// doi.org/10.1111/jar.12022

Gilrane-Mcgarry, U. y Taggart, L. (2012). An exploration of the support received by people with intellectual disabilities who have been bereaved. Journal of Research in Nursing, 12, 129-144. https://doi.org/10.1177/1744987106075611

Hollins, S. y EsterhuYZen, A. (1997). Bereavement and grief in adults with intellectual disabilities. British Journal of Psychiatry, 170, 497-501. https://doi.org/10.1192/bjp.170.6.497

Hume, K., Regan, T., Megronigle, L. y Rhinehalt, C. (2016). Supporting students with autism spectrum disorder through grief and loss. Teaching Exceptional Children, 48(3), 128136. https://doi.org/10.1177/0040059915618196

*Irwin, L., O’Malley, G., Neelofur, S. y Guerin, S. (2017). An exploration of clinical psychology's response to parental bereavement in adults with intellectual disability. Journal of Applied Research in Intellectual Disabilities, 30(6), 1065-1075. https://doi.org/10.1111/ jar. 12380

James, I. A. (1995). Helping people with learning disability to cope with bereavement. British Journal of Learning Disabilities, 23, 74-78. https://doi.org/10.1111/j.1468-3156.1995. tb00168.x

Kitching, N. (1987). Helping people with mental handicaps cope with bereavement: a case study with discussion. British Journal of Learning Disabilities, 15(2), 48-86. https://doi. org/10.1111/j.1468-3156.1987.tb00395.x

Koemler, K. (2016). Supporting children and young people with Autism Spectrum Disorder through bereavement. Bereavement Care, 35(3), 94-101. https://doi.org/10.1080/0268262 1.2016.1254437

LAWHON, T. (2004). Teachers and schools can aid grieving students. Education, 124(3), 559-566.

Lord, A. J., FIELD, S. y SMITH, I. C. (2017). The experiences of staff who support people with intellectual disability on issues about death, dying and bereavement: a metasynthesis. Journal 
FACTORES DETERMINANTES DEL DUELO EN PERSONAS

CON DISCAPACIDAD INTELECTUAL Y TEA: REVISIÓN SISTEMÁTICA

M. BÓVEDA HERMOSILLA Y N. FLORES ROBAINA

of Applied Research in Intellectual Disabilities, 30(6), 1007-1021. https://doi.org/10.1111/ jar.12376

Machale, R. y Carey, S. (2002). An investigation of the effects of bereavement on mental health and challenging behaviour in adults with learning disability. British Journal of Learning Disabilities, 30(3), 113-117. https://doi.org/10.1046/j.1468-3156.2002.00166.x

Machale, R., Mcevoy, J. y Tierney, E. (2009). Caregiver perceptions of the understanding of death and need for bereavement support in adults with intellectual disabilities. Journal of Applied Research in Intellectual Disabilities, 22(6), 574-581. https://doi.org/10.1111/j.14683148.2009.00513.x

Mappin, R. y Hanlon, D. (2005). Description and evaluation of a bereavement group for people with learning disabilities. British Journal of Learning Disabilities, 33(3), 106-112. https://doi.org/10.1111/j.1468-3156.2005.00339.x

Mcadams, E. y STOUgh, L. (2018). Teacher perspectives on grief among children with intellectual disabilities. Journal of Loss and Trauma, 23(2), 159-175. https://doi.org/10.1080/1532 5024.2018.1434859

Mcbride, J. y Simms, S. (2001). Death in the family: adapting a family systems framework to the grief process. American Journal of Family Therapy, 29(1), 59-73. https://doi.org/ $10.1080 / 019261801750182414$

McCleAn, K. y Guerin, S. (2019). A qualitative analysis of psychologists' views of bereavement among children with intellectual disability in Ireland. British Journal of Learning Disabilities, 47(4), 247-254. https://doi.org/ 10.1111/bld.12284

Mcevoy, J., Machale, R. y Tierney, E. (2012). Concept of death and perceptions of bereavement in adults with intellectual disabilities. Journal of Intellectual Disability Research, 56(2), 191-203. https://doi.org/10.1111/j.1365-2788.2011.01456.x

*Mcevoy, J., Treacy, B. y Quigley, J. (2017). A matter of life and death: knowledge about the body and concept of death in adults with intellectual disabilities. Journal of Intellectual Disability Research, 61(1), 89-98. https://doi.org/10.1111/jir.12347

*Mcritchie, R., Mckenzie, K., Quayle, E., Harlin, M. y Neumann, K. (2014). How adults with an intellectual disability experience bereavement and grief: a qualitative exploration. Death Studies, 38(3), 179-185. https://doi.org/10.1080/07481187.2012.738772

Melhem, N. M., Moritz, G., Walker, M., Shear, M. K. y Brent, D. (2007). Phenomenology and correlates of complicated grief in children and adolescents. Journal of the American Academy of Child and Adolescent Psychiatry, 46, 493-499. https://doi.org/10.1097/ chi.Ob013e31803062a9

Mesibov, G. B., Shea, V. y Schopler, E. (2005). The Teacch approach to autism spectrum disorders. Springer Science \& Business Media.

Muñiz, V., Alcedo, M. A. y Gómez, L. E. (2017). El proceso de duelo de personas con discapacidad intelectual. Siglo Cero, 48(3), 7-25. https://doi.org/14201/scero2017483725

*O’Keeffe, L., Guerin, S., Mcevoy, J., Lockhart, K. y Dodd, P. (2019). The process of developing self-report measures in intellectual disability: a case study of a complicated grief scale. British Journal of Learning Disabilities, 47(2), 134-144. https://doi.org/10.1111/ bld.12261

Oswin, M. (1992). Don't ask us to dance: some aspects of bereavement for people who have learning difficulties. Clinical Psycholy Forum, 44, 16-21.

Rodríguez-Herrero, P., De la Herrán, A. e Izuzquiza, D. (2013). “Y si me muero... ¿ ¿ónde está mi futuro?” Hacia una educación para la muerte en personas con discapacidad intelectual. Educación XXI, 16(1), 329-350. https://doi.org/10.5944/educxx1.16.1.729 


\section{FACTORES DETERMINANTES DEL DUELO EN PERSONAS CON DISCAPACIDAD INTELECTUAL Y TEA: REVISIÓN SISTEMÁTICA M. BÓVEDA HERMOSILLA Y N. FLORES ROBAINA}

*Rodríguez-Herrero, P., Izuzquiza, D. y De la Herrán, A. (2013). Diseño, aplicación y evaluación de un programa de educación para la muerte dirigido a personas adultas con discapacidad intelectual. Revista Iberoamericana de Educación, 63(1), 199-219.

*Rodríguez-Herrero, P., Izuzquiza, D. y De la Herrán, A. (2015). Concept of death in young people with intellectual disability: a contribution to the pedagogy on death. Siglo Cero, 46(1), 67-80. https://doi.org/10.14201/scero20154616780

*Stancliffe, R. J., Wiese, M. Y., Read, S., Jeltes, G. y Clayton, J. M. (2016). Knowing, planning for and fearing death: do adults with intellectual disability and disability staff differ? Research in Developmental Disabilities, 49, 47-59. https://doi.org/10.1016/j. ridd.2015.11.016

Stoddart, K. P., Burke, L. y Temple, V. (2002). Outcome evaluation of bereavement groups for adults with intellectual disabilities. Journal of Applied Research in Intellectual Disabilities, 15(1), 28-35. https://doi.org/ 10.1046/j.1468-3148.2002.00084.x

*Thorp, N., Stedmon, J. y Lloyd, H. (2018). "I carry her in my heart": an exploration of the experience of bereavement for people with learning disability. British Journal of Learning Disabilities, 46(1), 45-53. https://doi.org/10.1111/bld.12212

TuFFrey-WiJne, I. y Rose, T. (2017). Investigating the factors that affect the communication of death-related bad news to people with intellectual disabilities by staff in residential and supported living services: an interview study. Journal of Intellectual Disability Research, 61(8), 727-736. https://doi.org/10.1111/jir.12375

WASs, H. y CORR, C. A. (1982). Helping children cope with death: guidelines and resources. Hemisphere Publishing Corporation.

Worden, J. W. (2013). El tratamiento del duelo. Asesoramiento psicológico y terapia. Paidós.

*Young, H. y Garrard, B. (2016). Bereavement and loss: developing a memory box to support a young woman with profound learning disabilities. British Journal of Learning Disabilities, 44(1), 78-84. https://doi.org/10.1111/jir.1237510.1111/bld.12129

*Zakreski, M. J. (2017). Treating complicated grief in a teenager with autism: when the client's limitations prevent engagement in therapy. Clinical Practice in Pediatric Psychology, 5(3), 273. https://doi.org/10.1111/jir.1237510.1037/cpp0000191 


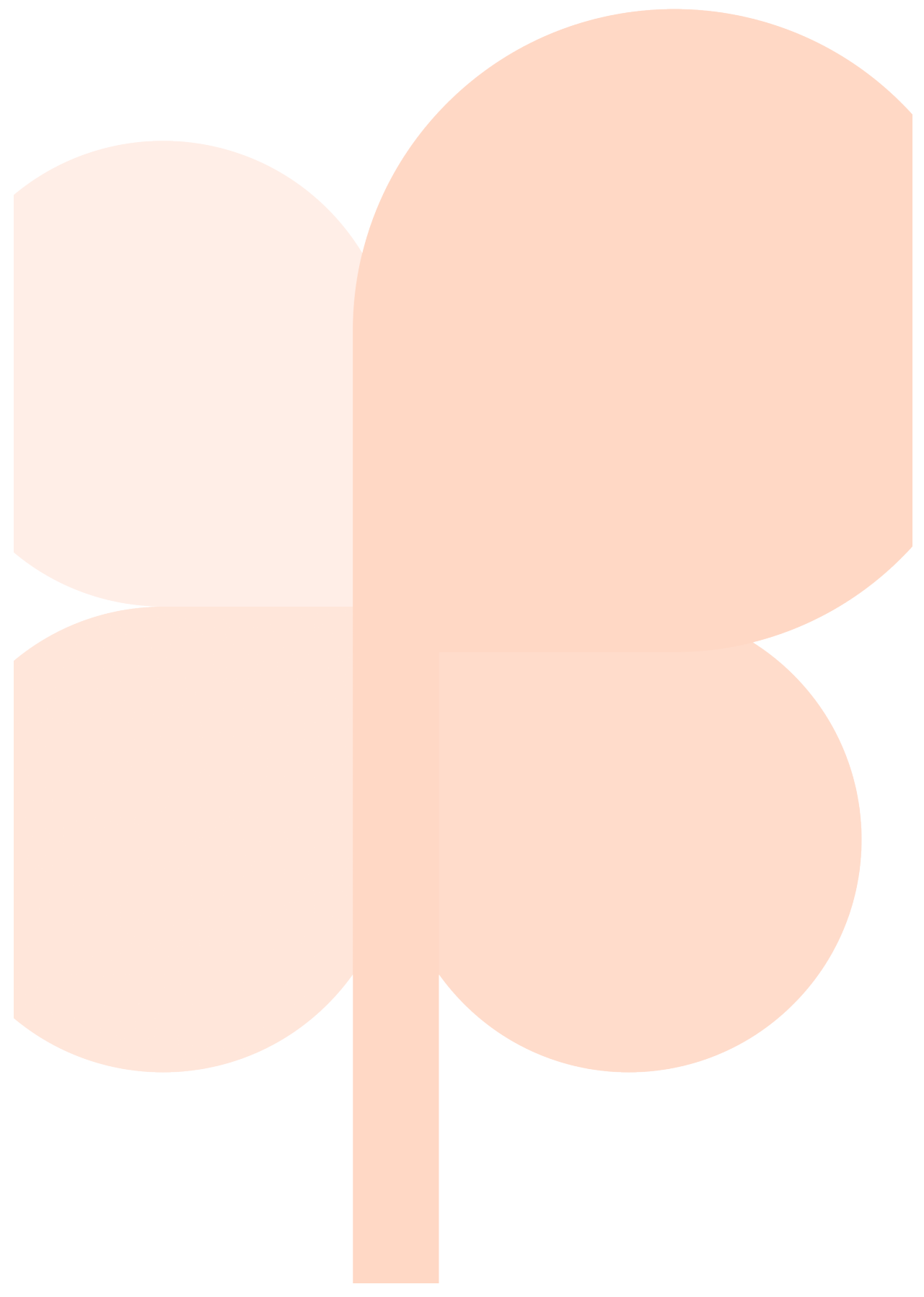

